Neural regulation of the stress response: glucocorticoid feedback mechanisms

J.P. Herman, J.M. McKlveen, M.B. Solomon, E. Carvalho-Netto and B. Myers

The Brazilian Journal of Medical and Biological Research is partially financed by

\section{贵CNPq} Conseliho Nacional de Des
cientifico e Tecrológico

Ministério da Ciência e Tecnologia

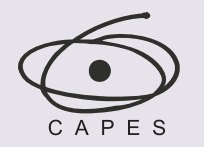

Ministério da Educação
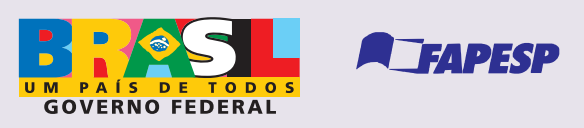

Institutional Sponsors

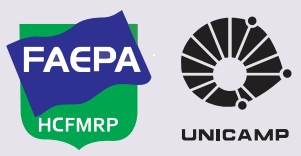

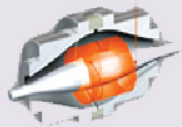

1DID Associaçāo Explore High - Performance MS Orbitrap Technology In Proteomics \& Metabolomics analitica $\underset{\text { analiticaweb.com.br }}{\text { Thermo }}$ SCI ENIFIC $\oplus$ SHIMADZU

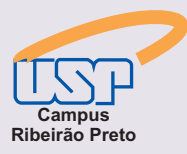




\title{
Neural regulation of the stress response: glucocorticoid feedback mechanisms
}

\author{
J.P. Herman ${ }^{1}$, J.M. McKlveen ${ }^{1}$, M.B. Solomon ${ }^{1}$, E. Carvalho-Netto ${ }^{2}$ and B. Myers ${ }^{1}$ \\ ${ }^{1}$ Department of Psychiatry and Behavioral Neuroscience, University of Cincinnati, Cincinnati, OH, USA \\ ${ }^{2}$ CAPES, Ministério da Educação, Brasília, DF, Brasil
}

\begin{abstract}
The mammalian stress response is an integrated physiological and psychological reaction to real or perceived adversity. Glucocorticoids are an important component of this response, acting to redistribute energy resources to both optimize survival in the face of challenge and to restore homeostasis after the immediate challenge has subsided. Release of glucocorticoids is mediated by the hypothalamo-pituitary-adrenal (HPA) axis, driven by a neural signal originating in the paraventricular nucleus (PVN). Stress levels of glucocorticoids bind to glucocorticoid receptors in multiple body compartments, including the brain, and consequently have wide-reaching actions. For this reason, glucocorticoids serve a vital function in negative feedback inhibition of their own secretion. Negative feedback inhibition is mediated by a diverse collection of mechanisms, including fast, non-genomic feedback at the level of the PVN, stress-shut-off at the level of the limbic system, and attenuation of ascending excitatory input through destabilization of mRNAs encoding neuropeptide drivers of the HPA axis. In addition, there is evidence that glucocorticoids participate in stress activation via feed-forward mechanisms at the level of the amygdala. Feedback deficits are associated with numerous disease states, underscoring the necessity for adequate control of glucocorticoid homeostasis. Thus, rather than having a single, defined feedback 'switch', control of the stress response requires a wide-reaching feedback 'network' that coordinates HPA activity to suit the overall needs of multiple body systems.
\end{abstract}

Key words: Hypothalamo-pituitary-adrenocortical axis; Corticotropin-releasing hormone; Glucocorticoid receptor; Amygdala; Hippocampus; Prefrontal cortex

\section{Endocrine stress responses: hypothalamo-pituitary-adrenocortical axis}

Appropriate responses to stress promote survival by altering physiological processes and behavior. The principal endocrine component of the stress response involves activation of the hypothalamo-pituitary-adrenocortical (HPA) axis, which involves a neuroendocrine cascade culminating in the synthesis and secretion of glucocorticoids (primarily cortisol in humans and primarily corticosterone in rats, mice and other species). The primary physiological action of glucocorticoid signaling is redistribution of energy, increasing availability of fuels in order to promote survival capacity in the face of real or perceived threats (i.e., stressors). The largely catabolic actions of glucocorticoids require tight control, lest the organism experience long-term changes in metabolic function that can have deleterious actions. Regulation of glucocorticoid secretion is therefore subject to feedback control by, as we shall see below, a diverse and situationally regulated array of glucocorticoid signaling mechanisms.

Activation of the HPA axis is controlled by a relatively small set of parvocellular neurons located in the paraventricular nucleus of the hypothalamus (PVN). Upon adequate stimulation by stress or circadian drive, these neurons release neural factors, such as corticotrophin-releasing hormone $(\mathrm{CRH})$ and arginine vasopressin (AVP), into the hypophyseal portal circulation. These factors then travel to the anterior pituitary and cause release of adrenocorticotrophic hormone (ACTH), which is released into the systemic circulation and causes synthesis and secretion of glucocorticoids by the adrenal cortex. Once released, glucocorticoids are able to bind high-affinity mineralocorticoid receptors (MR) or lower-affinity glucocorticoid receptors (GRs), which function as ligand-gated transcription factors to positively or negatively regulate gene expression (believed to be a primary glucocorticoid effect on homeostasis) (1). The affinity of the MR causes them to be extensively bound at low

Correspondence: J.P. Herman, Metabolic Diseases Institute, University of Cincinnati, 2170 E. Galbraith Rd, Cincinnati,

OH 45237-0506, USA. Fax: +1-513-558-9104. E-mail: james.herman@uc.edu

Presented at the III Fórum em Neurobiologia do Estresse, Araraquara, SP, Brazil, September 8-10, 2011.

Received January 14, 2012. Accepted March 9, 2012. Available online March 30, 2012. Published April 9, 2012. 
circulating levels of glucocorticoids, and they are thought to be important in ambient glucocorticoid signaling processes (e.g., controlling basal secretion across the circadian cycle) (2). In contrast, the GR is bound only during periods of high circulating glucocorticoid levels, as experienced during stress. Given this binding profile, it is not surprising to learn that the majority of glucocorticoid negative feedback mechanisms are mediated by GRs (1).

The ability for glucocorticoids to close the feedback loop via the GRs makes for a logical and compelling mechanism for control of stress responses. However, as we shall see, glucocorticoid signaling has several nuances that lend both redundancy and complexity to the HPA regulatory process.

\section{Glucocorticoid 'fast' feedback: non-genomic mechanisms}

Given the traditional view that glucocorticoids work by changing gene transcription, it is always surprising to learn of the relative speed by which negative feedback occurs. From landmark work beginning in the 1960s, we know that the most powerful glucocorticoid inhibition of the HPA axis occurs within minutes $(3,4)$. This time-frame is far too fast to be mediated by genomic effects and occurs at the level of the CNS, an assumption proven by Keller-Wood and Dallman (5). Thus, rapid feedback must be mediated by non-genomic actions, perhaps working at or near the cell membrane.

The mechanism underlying fast feedback remained enigmatic until only recently. Elegant electrophysiologic studies indicate that glucocorticoids bind membrane receptors on PVN CRH neurons, eliciting an intracellular cascade that mobilizes the synthesis of endocannabinoids (Figure 1). Endocannabinoid release then causes presynaptic inhibition of glutamate release, which reduces the neural activity of parvocellular neurons (6). Our group has since evaluated this mechanism in vivo, and documented that local injection of a cannabinoid receptor 1 antagonist can block negative feedback inhibition of ACTH and corticosterone release (7). Local infusion of a membrane-impermeant glucocorticoid conjugate (dexamethasone bound to bovine serum albumin (BSA)) is equally effective as an unconjugated steroid in inhibiting HPA axis stress responses, suggesting that fast feedback effects are mediated by glucocorticoid binding at or near the membrane (7).

The receptor mediating fast feedback has yet to be identified. Research by Moore's group (8) suggests the existence of a G-protein-coupled 'membrane GR' (mGR) that is responsible for the fast effects of glucocorticoids on behavior (e.g., the clasping reflex in the newt) (8). A membrane-bound receptor was also proposed by Di et al. (6), based on evidence that glucocorticoid fast feedback was not blocked by MR or GR antagonists. However, the GR antagonist used in their study (mifepristone) causes nuclear translocation of the GR and may be specific for genomic mechanisms of receptor signaling (9), leaving a 'membrane' action of the traditional GR open to possibility. Indeed, recent research suggests that mice bearing GR deletion in the PVN (targeted by an Sim1 promoter-cre recombinase driver crossed with mice bearing GRflox alleles) do not exhibit rapid inhibition of PVN neuronal excitation in vitro (10), suggesting the possibility that fast feedback is indeed mediated by the classical GR. In support of this hypothesis, electron microscopy studies have documented localization of GR immunoreactivity to the cell membrane $(11,12)$. Nonetheless, at present, the true identity of the membrane receptor remains to be determined.

\section{Neural inhibition of stress responses: role of limbic structures}

A rich literature ascribes feedback control of the HPA axis stress responses to limbic system structures, including the hippocampus and the medial prefrontal cortex (Figure 1) (see Refs. 13-15). Electrical stimulation of the hippocampus is sufficient to reduce circulating glucocorticoid levels, consistent with an inhibitory effect of hippocampal activation on the HPA axis (14). Inhibition of the stress responses of the HPA axis is also observed following chemical stimulation of the prelimbic division of the prefrontal cortex (16). Lesion studies indicate that destruction of the hippocampus (particularly its primary ventral output, the ventral subiculum) prolongs HPA axis responses to acute stressors of a psychogenic nature (i.e., stressors that signal potential, rather than emergent threats to homeostasis) (17). More recently, similar findings have been reported for the medial prefrontal cortex, where lesions delay shut-off of HPA axis responses to psychogenic stress (18-20). In both cases, the inhibitory mechanism requires an intermediary synapse to translate glutamatergic output into GABAergic inhibition at the PVN $(14,15)$. The timing of response prolongation following either prefrontal or hippocampal damage is $1-2 \mathrm{~h}$ poststress, suggesting a probable genomic mechanism.

Both the hippocampus and prefrontal cortex richly express GR (21), suggesting that inhibition of stress responses by these regions is mediated by glucocorticoid feedback. In the case of the prefrontal cortex, local implants of corticosterone are sufficient to reduce HPA axis responses to psychogenic stress, but not to stressors that signal a true physiologic threat (e.g., respiratory challenge caused by ether inhalation) (18). Evidence for local glucocorticoid regulation of hippocampal inhibition is less well established (possibly due to the difficulty in locally administering glucocorticoids across a large and elongated structure).

The role of the GR in limbic feedback regulation of the HPA axis was addressed in a series of studies using mice bearing deletion of the GR in the limbic forebrain (FBGRKO 
mice) (using a CamKII alpha promoter-cre recombinase driver mouse crossed with mice bearing GRflox alleles). The FBGRKO mouse has GR deleted in the cerebral cortex, hippocampus and basolateral amygdala, but retains GR expression in regions such as the central and medial amygdaloid nuclei and, importantly, the PVN (as well as in all peripheral tissues) $(22,23)$. Stress testing reveals that FBGRKO mice have delayed shut-off of HPA axis responses to psychogenic, but not systemic stressors, and are deficient in dexamethasone suppression of the circadian corticosterone peak $(22,23)$. While these mice cannot distinguish between actions at the prefrontal cortex vs the hippocampus, the data provide evidence for limbic forebrain GR control of negative feedback inhibition of the HPA axis. Importantly, the data also indicate that forebrain GR feedback is stressor-specific: glucocorticoid inhibition of the HPA axis is only seen when the stressful stimulus actively engages the brain structure(s) in question.

The relative contribution of the hippocampus vs prefrontal cortex was recently addressed by Radley and Sawchenko (24). Reasoning that the hippocampus and prefrontal cortex are interconnected, these investigators

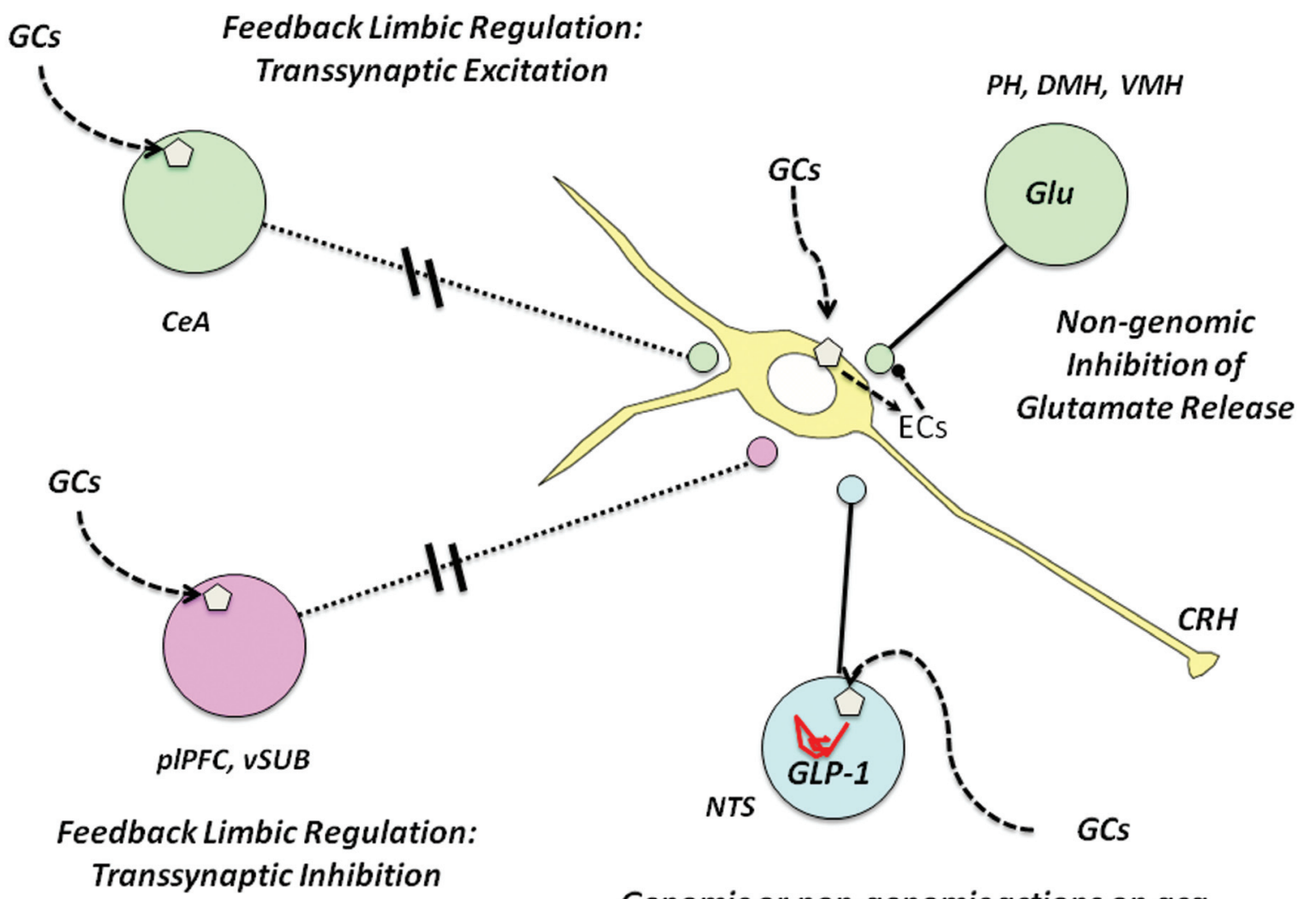

Genomic or non-genomic actions on gcg
mRNA Stability

Figure 1. Glucocorticoid (GC) signaling mechanisms regulating paraventricular nucleus (PVN) corticorticotropin-releasing hormone $(\mathrm{CRH})$ neurons. Glucocorticoid negative feedback can generally be divided into three interacting domains. GCs provide rapid, nongenomic inhibition at the PVN, mediated by endocannabinoid (EC) inhibition of PVN glutamate inputs from regions such as the posterior hypothalamus $(\mathrm{PH})$, dorsomedial hypothalamus $(\mathrm{DMH})$ and ventromedial hypothalamus ( $\mathrm{VMH}$; upper right). Forebrain genomic $\mathrm{GC}$ signaling is also a key component of feedback regulation, mediated via the prelimbic division of the prefrontal cortex (pIPFC) and ventral subiculum (vSUB). These structures have little or no direct interactions with the PVN and require intermediary synapses in PVN-projecting cell groups (broken lines). Glucocorticoids act on limbic output circuits (indicated in red) to inhibit the PVN (lower left). Glucocorticoids appear to act by yet a third mechanism to destabilize mRNAs (preproglucagon, gcg) encoding HPA-activating neuropeptides such as glucagon-like peptide-1 (GLP-1), thereby removing excitatory input to the PVN (lower right). Finally, GCs may also play a role in stress excitation, mediated by transsynaptic inputs from regions such as the central amygdaloid nucleus (CeA; upper left). NTS = nucleus tractus solitarii. 
used lesion and anatomical studies to determine whether these structures controlled HPA axis function in series or in parallel. The data indicate that the prefrontal cortex and hippocampus innervate common subcortical targets, and that combined lesions had additive effects on HPA axis hyperactivity, suggesting that both regions are involved in the regulatory process, likely acting in parallel.

\section{Ascending feedback mechanisms: inhibition by subtraction?}

Feedback control of stressors signaling emergent homeostatic challenges is not well understood. In fact, KellerWood and Dallman (5) suggest that some stimuli, such as ether exposure, may not be subject to direct feedback, perhaps due to the necessity of glucocorticoids for meeting systemic challenges. Drive of the HPA axis by homeostatic imbalance takes several forms: sensing peripheral chemical imbalances by circumventricular organs (e.g., elevated angiotensin II in the subfornical organ, elevated circulating cytokines in the area postrema); sensing changes in metabolism via altered leptin/insulin signaling in the mediobasal hypothalamus, and relaying neural signals from the lungs, heart, gut and other internal organs via visceral sensory afferents (14). The latter mechanism prominently involves noradrenergic as well as non-catecholaminergic relay neurons in the nucleus tractus solitarii (NTS), which sends direct excitatory projections to $\mathrm{CRH}$ neurons of the PVN (15). Importantly, the NTS receives information from limbic regions as well as the periphery, and may be a site of general stress response coordination (15).

Neurons of the NTS express GR, and are thus plausible targets for glucocorticoid modulation of HPA axis responses (25). Currently, data directly exploring NTS glucocorticoid feedback and HPA axis function are lacking. However, recent research from our group demonstrates that expression of the preproglucagon $(g c g)$ gene, which encodes the HPA-excitatory neuropeptide glucagon-like peptide 1 , is rapidly down-regulated by stress-induced glucocorticoid secretion (26). Down-regulation of gcg expression occurs within 30 min of stress or glucocorticoid exposure, and is independent of effects on gene transcription. These data suggest that effects of stress and glucocorticoids are nongenomic, probably mediated by gcg mRNA destabilization and degradation. Importantly, loss of gcg gene expression in NTS is accompanied by a stress-induced depletion of the GLP-1 peptide in the PVN, suggesting reduced capacity for PVN excitation by a subsequent stressor (26). Loss of a potential excitatory peptidergic species is reminiscent of the stress-refractory period seen in the hours following acute stress, wherein subsequent stimulation cannot induce an HPA axis stress responses (see Ref. 5). While ascribing a role for the brainstem in stress refractivity is speculative, the data suggest that glucocorticoids modulate the capacity of excitatory input to the PVN via a non-genomic mechanism targeting RNA stability, a mechanism distinct from either genomic modulation or membrane signaling.

\section{The other side of feedback: feed-forward mechanisms?}

To this point, we have focused largely on inhibitory effects of glucocorticoids on HPA axis activity. However, it is clear that there is another side to glucocorticoid signaling in the brain, wherein stress-excitatory regions, such as the amygdala, may be activated by the hormone. For example, stress exposure promotes $\mathrm{CRH}$ release in the central amygdala (27), which is linked to enhanced fear and anxiety. Stress-induced release of $\mathrm{CRH}$ is blocked by the GR antagonist mifepristone, indicating a role for the GR in amygdalar $\mathrm{CRH}$ secretion in response to stressors (28). Notably, the central amygdaloid nucleus is linked to excitation of the HPA axis, particularly following stimuli signaling homeostatic disruption (29). Region-specific knockdown of the GR in the central amygdaloid region impairs fear conditioning and reduces HPA axis responses during cued recall testing, suggesting that glucocorticoid signaling is vital for the integration of fear memories and related HPA drive (30). While the role for the amygdaloid GR in acute feed forward regulation of the HPA axis remains to be established, these data suggest that glucocorticoids may be involved in generation as well as inhibition of glucocorticoid release.

Chronic glucocorticoid exposure and chronic stress increase expression of CRH mRNA in the central amygdala $(31,32)$, suggesting that these neurons may be 'recruited' during chronic stress and promote development of chronic stress-related pathologies. Notably, the central amygdaloid nucleus is among a short list of brain regions that show enhanced activation by an acute stress after chronic stress exposure, suggesting a role in stress sensitization. Together, these data suggest that feed-forward actions of glucocorticoids may be involved in the generation of pathological responses in the context of chronic stress.

\section{Dynamic regulation of feedback mechanisms}

Negative feedback regulation of the HPA axis is subject to regulation via any number of mechanisms, most of which affect GR function. Animal studies link deficits in negative feedback to reduced glucocorticoid signaling in limbic regions such as the hippocampus. For example, chronic stress paradigms that generate HPAaxis hyperactivity and deficits in dexamethasone suppressing are associated with reduced GR expression in the hippocampus and prefrontal cortex (33-35). Early life manipulations (maternal deprivation) cause lower GR expression in hippocampus and cortex, resulting in prolonged responses to stress and deficient glucocorticoid feedback (36). Finally, forebrain deletion of the GR (see above) inhibits dexamethasone suppression of the HPA axis (22). 
There are also conditions that enhance feedback efficacy. Early life handling and/or attentive maternal care increases GR expression in rodents and reduces HPA axis stress responses later in life, phenomena linked to epigenetic modification of the GR promoter (37). In addition, recovery from chronic variable stress exposure generates a period of reduced HPA axis reactivity to acute stress (38), suggesting a compensatory enhancement of feedback regulation of the HPA axis. However, behavioral studies note impaired extinction and exaggerated reinstatement of conditioned fear during 'recovery' from chronic variable stress (39), effects suggesting long-term pathological changes in stress reactivity. The relationship of behavioral deficits to attenuated corticosterone responses remains to be determined.

\section{Feedback and pathology}

Glucocorticoid dyshomeostasis is heavily represented in disease states, including stress-related disorders such as depression and post-traumatic stress disorder (PTSD). The nature of glucocorticoid deficits varies among diseases; for example, in the case of (melancholic) depression, glucocorticoid levels tend to be elevated, whereas PTSD is linked to pathologically low glucocorticoid levels (see Refs. 40,41).

Glucocorticoid deficits in both depression and PTSD are linked to altered negative feedback efficacy. In the case of depression, a sizable subpopulation of patients exhibit deficient glucocorticoid feedback inhibition of the HPA axis, manifest as 'escape' from dexamethasone suppression of the circadian cortisol rise (e.g., Refs. 42,43). Deficits in feedback resemble those seen in animal models of chronic stress, maternal deprivation or limbic GR knock-down $(34,36,37)$. Of note, all of the aforementioned manipulations increase depression-like behavior $(34,36,37)$. In humans, reduced size of the anterior cingulate cortex (a division of the human prefrontal complex) correlates with impaired dexamethasone suppression, suggesting that a link between limbic cortex and HPA axis feedback efficacy

\section{References}

1. de Kloet ER, Vreugdenhil E, Oitzl MS, Joels M. Brain corticosteroid receptor balance in health and disease. Endocr Rev 1998; 19: 269-301.

2. Dallman MF, Levin N, Cascio CS, Akana SF, Jacobson L, Kuhn RW. Pharmacological evidence that the inhibition of diurnal adrenocorticotropin secretion by corticosteroids is mediated via type I corticosterone-preferring receptors. Endocrinology 1989; 124: 2844-2850.

3. Dallman MF. Fast glucocorticoid actions on brain: back to the future. Front Neuroendocrinol 2005; 26: 103-108.

4. Tasker JG, Herman JP. Mechanisms of rapid glucocorticoid feedback inhibition of the hypothalamic-pituitary-adrenal also exists in man (44).

Reduced cortisol levels seen in PTSD patients are associated with the opposite phenomenon, i.e., enhanced glucocorticoid negative feedback (45). Patients with PTSD generally exhibit enhanced dexamethasone suppression of the HPA axis and lower basal cortisol levels. Recent data suggest that low cortisol levels may predispose individuals to the development of PTSD. Moreover, maternal PTSD is linked to lower cortisol levels in the offspring, perhaps placing individuals at a transgenerational 'risk' for developing the disorder (see Ref. 45). Of note, CSF CRH is elevated in PTSD, raising the possibility that enhanced feed-forward mechanisms may also be engaged in the disorder (46).

\section{Conclusions}

Glucocorticoid feedback regulation is a dynamic process that takes full advantage of the complexities of glucocorticoid signaling to provide a diverse and multi-faceted means of controlling stress reactivity. Inhibition of the HPA axis uses glucocorticoid signaling to 1) provide rapid shut-off of initiated responses at the cell membrane; 2) control response duration at the level of the limbic forebrain, and 3) provide long-latency attenuation of excitatory input by modulation of RNA stability. In addition to its importance in controlling day-to-day glucocorticoid exposure, feedback appears to play an important role in brain homeostasis, as deficits in glucocorticoid control have deleterious effects on mood and cognition in animal models and are cardinal endophenotypes in human diseases such as depression and PTSD. Understanding the nature of feedback control and feedback pathologies will be key to unlocking mechanisms of, and developing therapies for, stress-related disease states.

\section{Acknowledgments}

Research supported by US National Institutes of Health (Grants \#MH049698, \#MH069725, \#MH069860, and \#MH066958). axis. Stress 2011; 14: 398-406.

5. Keller-Wood ME, Dallman MF. Corticosteroid inhibition of ACTH secretion. Endocr Rev 1984; 5: 1-24.

6. Di S, Malcher-Lopes R, Halmos KC, Tasker JG. Nongenomic glucocorticoid inhibition via endocannabinoid release in the hypothalamus: a fast feedback mechanism. $J$ Neurosci 2003; 23: 4850-4857.

7. Evanson NK, Tasker JG, Hill MN, Hillard CJ, Herman JP. Fast feedback inhibition of the HPA axis by glucocorticoids is mediated by endocannabinoid signaling. Endocrinology 2010; 151: 4811-4819.

8. Orchinik M, Murray TF, Moore FL. A corticosteroid receptor 
in neuronal membranes. Science 1991; 252: 1848-1851.

9. Spiga F, Knight DM, Droste SK, Conway-Campbell B, Kershaw Y, MacSweeney CP, et al. Differential effect of glucocorticoid receptor antagonists on glucocorticoid receptor nuclear translocation and DNA binding. J Psychopharmacol 2011; 25: 211-221.

10. Haam J, Halmos KC, Muglia LJ, Tasker JG. Rapid synaptic modulation of hypothalamic neurons by glucocorticoids requires the glucocorticoid receptor. San Diego: Society for Neuroscience. Program No. 389.19; 2010.

11. Johnson LR, Farb C, Morrison JH, McEwen BS, LeDoux JE. Localization of glucocorticoid receptors at postsynaptic membranes in the lateral amygdala. Neuroscience 2005; 136: 289-299.

12. Liposits Z, Bohn MC. Association of glucocorticoid receptor immunoreactivity with cell membrane and transport vesicles in hippocampal and hypothalamic neurons of the rat. $\mathrm{J} \mathrm{Neu}$ rosci Res 1993; 35: 14-19.

13. Jacobson L, Sapolsky R. The role of the hippocampus in feedback regulation of the hypothalamic-pituitary-adrenocortical axis. Endocr Rev 1991; 12: 118-134.

14. Herman JP, Figueiredo $H$, Mueller NK, Ulrich-Lai $Y$, Ostrander MM, Choi DC, et al. Central mechanisms of stress integration: hierarchical circuitry controlling hypothalamopituitary-adrenocortical responsiveness. Front Neuroendocrinol 2003; 24: 151-180.

15. Ulrich-Lai YM, Herman JP. Neural regulation of endocrine and autonomic stress responses. Nat Rev Neurosci 2009; 10: 397-409.

16. Jones KR, Myers B, Herman JP. Stimulation of the prelimbic cortex differentially modulates neuroendocrine responses to psychogenic and systemic stressors. Physiol Behav 2011; 104: 266-271.

17. Herman JP, Dolgas CM, Carlson SL. Ventral subiculum regulates hypothalamo-pituitary-adrenocortical and behavioural responses to cognitive stressors. Neuroscience 1998; 86: 449-459.

18. Diorio D, Viau V, Meaney MJ. The role of the medial prefrontal cortex (cingulate gyrus) in the regulation of hypothalamicpituitary-adrenal responses to stress. J Neurosci 1993; 13: 3839-3847.

19. Figueiredo HF, Bruestle A, Bodie B, Dolgas CM, Herman JP. The medial prefrontal cortex differentially regulates stressinduced c-fos expression in the forebrain depending on type of stressor. Eur J Neurosci 2003; 18: 2357-2364.

20. Radley JJ, Sawchenko PE. Differential role of the dorsal versus ventral medial prefrontal cortex (mPFC) in paraventricular hypothalamic responses to acute restraint stress. Washington: Society for Neuroscience. Program No. 637.11; 2005.

21. Herman JP. Regulation of adrenocorticosteroid receptor mRNA expression in the central nervous system. Cell Mol Neurobiol 1993; 13: 349-372.

22. Boyle MP, Brewer JA, Funatsu M, Wozniak DF, Tsien JZ, Izumi $Y$, et al. Acquired deficit of forebrain glucocorticoid receptor produces depression-like changes in adrenal axis regulation and behavior. Proc Natl Acad Sci U S A 2005; 102: 473-478.

23. Furay AR, Bruestle AE, Herman JP. The role of the forebrain glucocorticoid receptor in acute and chronic stress. Endocrinology 2008; 149: 5482-5490.

24. Radley JJ, Sawchenko PE. A common substrate for prefron- tal and hippocampal inhibition of the neuroendocrine stress response. J Neurosci 2011; 31: 9683-9695.

25. Sawchenko PE, Bohn MC. Glucocorticoid receptor-immunoreactivity in $\mathrm{C} 1, \mathrm{C} 2$, and $\mathrm{C} 3$ adrenergic neurons that project to the hypothalamus or to the spinal cord in the rat. J Comp Neurol 1989; 285: 107-116.

26. Zhang R, Packard BA, Tauchi M, D'Alessio DA, Herman JP. Glucocorticoid regulation of preproglucagon transcription and RNA stability during stress. Proc Natl Acad Sci U S A 2009; 106: 5913-5918.

27. Cook CJ. Stress induces CRF release in the paraventricular nucleus, and both CRF and GABA release in the amygdala. Physiol Behav 2004; 82: 751-762.

28. Cook CJ. Glucocorticoid feedback increases the sensitivity of the limbic system to stress. Physiol Behav 2002; 75: 455464.

29. Xu Y, Day TA, Buller KM. The central amygdala modulates hypothalamic-pituitary-adrenal axis responses to systemic interleukin-1beta administration. Neuroscience 1999; 94: 175-183.

30. Kolber BJ, Roberts MS, Howell MP, Wozniak DF, Sands MS, Muglia LJ. Central amygdala glucocorticoid receptor action promotes fear-associated $\mathrm{CRH}$ activation and conditioning. Proc Natl Acad Sci U S A 2008; 105: 12004-12009.

31. Makino S, Gold PW, Schulkin J. Corticosterone effects on corticotropin-releasing hormone mRNA in the central nucleus of the amygdala and the parvocellular region of the paraventricular nucleus of the hypothalamus. Brain Res 1994; 640: 105-112.

32. Makino S, Shibasaki T, Yamauchi N, Nishioka T, Mimoto T, Wakabayashi I, et al. Psychological stress increased corticotropin-releasing hormone mRNA and content in the central nucleus of the amygdala but not in the hypothalamic paraventricular nucleus in the rat. Brain Res 1999; 850: 136143.

33. Herman JP, Adams D, Prewitt C. Regulatory changes in neuroendocrine stress-integrative circuitry produced by a variable stress paradigm. Neuroendocrinology 1995; 61: 180-190.

34. Mizoguchi K, Ishige A, Aburada M, Tabira T. Chronic stress attenuates glucocorticoid negative feedback: involvement of the prefrontal cortex and hippocampus. Neuroscience 2003; 119: 887-897.

35. Sapolsky RM, Krey LC, McEwen BS. Stress down-regulates corticosterone receptors in a site-specific manner in the brain. Endocrinology 1984; 114: 287-292.

36. Ladd CO, Huot RL, Thrivikraman KV, Nemeroff CB, Plotsky $\mathrm{PM}$. Long-term adaptations in glucocorticoid receptor and mineralocorticoid receptor mRNA and negative feedback on the hypothalamo-pituitary-adrenal axis following neonatal maternal separation. Biol Psychiatry 2004; 55: 367-375.

37. Weaver IC, Cervoni N, Champagne FA, D'Alessio AC, Sharma S, Seckl JR, et al. Epigenetic programming by maternal behavior. Nat Neurosci 2004; 7: 847-854.

38. Ostrander MM, Ulrich-Lai YM, Choi DC, Richtand NM, Herman JP. Hypoactivity of the hypothalamo-pituitary-adrenocortical axis during recovery from chronic variable stress. Endocrinology 2006; 147: 2008-2017.

39. McGuire J, Herman JP, Horn PS, Sallee FR, Sah R. Enhanced fear recall and emotional arousal in rats recovering from chronic variable stress. Physiol Behav 2010; 101: 474482. 
40. Gold PW, Chrousos GP. Organization of the stress system and its dysregulation in melancholic and atypical depression: high vs low $\mathrm{CRH} / \mathrm{NE}$ states. Mol Psychiatry 2002; 7 : 254-275.

41. Yehuda R, Giller EL, Southwick SM, Lowy MT, Mason JW. Hypothalamic-pituitary-adrenal dysfunction in posttraumatic stress disorder. Biol Psychiatry 1991; 30: 1031-1048.

42. Carroll, Curtis GC, Mendels J. Neuroendocrine regulation in depression. I. Limbic system-adrenocortical dysfunction. Arch Gen Psychiatry 1976; 33: 1039-1044.

43. Carroll BJ, Curtis GC, Mendels J. Neuroendocrine regulation in depression. II. Discrimination of depressed from nondepressed patients. Arch Gen Psychiatry 1976; 33: 1051-1058.
44. MacLullich AM, Ferguson KJ, Wardlaw JM, Starr JM, Deary IJ, Seckl JR. Smaller left anterior cingulate cortex volumes are associated with impaired hypothalamic-pituitary-adrenal axis regulation in healthy elderly men. J Clin Endocrinol Metab 2006; 91: 1591-1594.

45. Radley JJ, Kabbaj M, Jacobson L, Heydendael W, Yehuda R, Herman JP. Stress risk factors and stress-related pathology: neuroplasticity, epigenetics and endophenotypes. Stress 2011; 14: 481-497.

46. Baker DG, West SA, Nicholson WE, Ekhator NN, Kasckow JW, Hill KK, et al. Serial CSF corticotropin-releasing hormone levels and adrenocortical activity in combat veterans with posttraumatic stress disorder. Am J Psychiatry 1999; 156: $585-588$. 